Article

\title{
Medication adherence of rheumatic heart disease patients: a cross-sectional study in Bangladesh
}

\author{
Abrar Wahab ${ }^{1,2}$, Md. Mahabub Ul Islam², Mehbuba Mehnoor Laboni ${ }^{3}$, Anisa Fatema ${ }^{4}$, Abeda Saleha Renesa ${ }^{2}$ \\ and Mohammad Morshad Alam ${ }^{1,5^{*}}$ \\ ${ }^{1}$ Graduex Research Group, Dhaka, Bangladesh \\ ${ }^{2}$ Department of Public Health, North South University, Dhaka-1229, Bangladesh \\ ${ }^{3}$ Rajshahi Medical College, Rajshahi 6000, Bangladesh \\ ${ }^{4}$ University Dental College and Hospital, 120/A, Outer Circular Rd, Dhaka 1217, Bangladesh \\ ${ }^{5}$ BD HSSP, The World Bank, Dhaka, Bangladesh
}

${ }^{*}$ Corresponding author: Mohammad Morshad Alam, DLI Consultant, Planning Wing, Ministry of Health and family Welfare, The World bank. Phone: +8801819192002; E-mail: mohammad.alam01@ northsouth.edu

Received: 04 August 2019/Accepted: 10 September 2019/ Published: 30 September 2019

\begin{abstract}
Rheumatic heart disease (RHD) is a significant public health problem and Non-adherence to treatment is an important and often unrecognized risk factor for cardiovascular mortality. The study aimed to assess self-reported medication adherence by 8-item Morisky Medication Adherence Scale (MMAS-8) among rheumatic heart disease patients and determine the associated factors. A cross-sectional study was carried out by applying a structured interview to Rheumatic heart disease patients aged 18 or higher in a tertiary hospital in Bangladesh. Among Rheumatic heart disease patients, $63.5 \%$ showed medium adherence, $36.5 \%$ showed low adherence to medication. Walking habit $(\mathrm{P}=0.000)$, exercise habit $(\mathrm{P}=0.000)$, smoking habit $(\mathrm{P}=0.000)$, and duration of RHD $(\mathrm{P}=0.005)$ found significantly associated with medication adherence. Multiple logistic regression analysis also revealed, don't have walking habit more than 10 minutes at a time (AOR=2.416, 95\% CI: 1.212-4.816), don't having exercise habit $(\mathrm{AOR}=2.420,95 \% \mathrm{CI}: 1.206-4.859)$, don't having habit of smoking (AOR=0.392, 95\% CI: $0.183-0.841$ ), duration of RHD for less than 15 (AOR=4.190, 95\% CI: 0.851 - 20.631) are independent predictors of adherence. Our study demonstrated medium to low medication adherence in the Morisky Medication Adherence Scale among rheumatic heart disease patients.
\end{abstract}

Keywords: rheumatic heart disease; MMAS-8; cross-sectional study; Bangladesh

\section{Introduction}

Rheumatic heart disease (RHD) is a significant public health problem and leading non-communicable diseases in low- and middle-income countries (LMICs) (Carapetis et al., 2005; Marijon et al., 2012). According to recent estimates, about 33 million people are now living with rheumatic heart disease and an additional 47 million going through asymptomatic damage of heart valves (Nulu et al., 2017). As of 2010 globally, it resulted in 345,000 deaths, and every year, there are about 1.4 million deaths in low- and middle-income countries (Karthikeyan et al., 2012; Paar et al., 2010).

Medication adherence is the measurement to which a patient is correctly following various medical advice and maintaining situations including self-care, self-directed exercises, medical equipment use, or therapy sessions (WHO, 2003). Persistence and adherence to drug treatment represent the main factors necessary to gain a significant reduction in mortality and morbidity and to improve the use of financial resources (Busse et al., 2010; Sokol et al., 2005; Wilke et al., 2011). According to the WHO, estimates that the cost of nonadherence to drug therapy amounts to 125 million euros per year in Europe (WHO, 2003).

Both healthcare provider and patient affect medication adherence, but a positive physician-patient relationship 
plays a vital role in improving adherence (APA Highlights Newsletter, n.d.; Retrieved from https://www.jemds.com/data_pdf/1_Dr manmohandesai pharmocology final.pdf). Several critical barriers to lower adherence has been demonstrated in previously conducted studies includes of modern medication regimens complexity, not understanding benefits of treatment, side effects, high price of medicine, poor satisfaction towards the treatment and lack of communication with doctor or lack of trust between a health care provider and patients (Berhe et al., 2017; Elliott and Marriott, 2009).

Much of the morbidity and mortality due to RHD can be prevented by existing therapies (Carapetis et al., 2013). Several old reports from developing countries have documented inadequate adherence to secondary prophylaxis and poor control of OAC therapy (Engelman et al., 2016; Oldgren et al., 2014).

Therefore, our present study was conducted to determine the self-reported medication adherence by validated Bengali version of 8-item Morisky Medication Adherence Scale (MMAS-8) among rheumatic heart disease patients and determine the associated factors.

\section{Materials and Methods}

\subsection{Study design, setting and period}

A hospital-based cross-sectional survey was carried out by applying a structured interview of Rheumatic heart disease patients. Adherence was to medication was measured by the eight-item Morisky Medication Adherence Scale (MMAS-8), translated into Bengali and validated for the present study (Morisky and Green, 1986).

The interviews occurred between November and December of 2017 at the National Center for the Control of Rheumatic Fever and Heart Diseases (NCCRF/HD), a selected tertiary level hospital in Dhaka, Bangladesh.

We selected patients aged 18 or higher with a confirmed diagnosis of Rheumatic heart disease and continuing medication, attended in the out-door of the NCCRF/HD.

\subsection{Sample size estimation}

Considering the study of Islam et al., where $20 \%$ of the RHD patients found therapeutic non-compliant, as well as the absolute accuracy of 5\% and confidence interval of $95 \%$, a sample of 232 individuals, was determined (Islam and Majumder, 2016). By taking 5\% more samples, our sample size became 244.

\subsection{Ethical approval}

The Ethics Review Committee of the Department of Public health, North South University approved the protocol for this study of. Written informed consent was taken from each of the participants during data collection.

\subsection{Data collection and analysis}

We adopted a purposive sampling method to select the study participants. The degree of adherence was determined according to the score resulting from the sum of all the correct answers; Scores: $>2=$ low adherence, 1 or $2=$ medium adherence, $0=$ high adherence (Morisky DE, Green LW, 1986). As we did not get any patientreported high adherence to medication, so patient adherence was dichotomized into two groups: low adherence versus medium adherence. Data were checked for completeness and consistency, entered, cleaned in Microsoft XL (version 2013) and analyzed using SPSS statistical package software (version 23).

To summarize data, descriptive statistics like frequencies and percentages were used. Logistic regression used to calculate adjusted odds ratio. We set the $\mathrm{P}$ values of less than 0.05 for Statistical significance.

\section{Results}

We had selected 244 patients, eight of whom declined to participate in the study, and finally, we were able to include 230 patients due to the incompleteness of interviews. The mean age of patients was 34.88 years $( \pm$ 11.966), with a minimum age of 18 and a maximum of 75 years, and the majority (56.5\%) of them were females. Most of the study participants (73.9\%) were married, and the majority (61.7\%) were of normal weight. $13.5 \%$ of the study participants were graduate, and $8.3 \%$ found illiterate.

On the other hand, $45.7 \%$ were of the patients were found to walk regularly for more than 10 minutes at a time. $40 \%$ of study participants do regular physical exercise other than walking. However, $34.3 \%$ of the study participants were found smoker. 
Asian J. Med. Biol. Res. 2019, 5 (3)

Table 1. Descriptive and chi-square analysis of characteristics of Rheumatic heart disease patients.

\begin{tabular}{|c|c|c|c|c|c|}
\hline \multirow[t]{2}{*}{ Variables } & \multirow[t]{2}{*}{ Category } & \multicolumn{2}{|c|}{ Medication adherence level } & \multirow[t]{2}{*}{ Chi-square } & \multirow[t]{2}{*}{ P-Value } \\
\hline & & Low adherence & Medium adherence & & \\
\hline \multirow[t]{2}{*}{ Age } & $\leq 40$ years & $59(25.7 \%)$ & $92(40.0 \%)$ & \multirow[t]{2}{*}{1.234} & \multirow[t]{2}{*}{0.267} \\
\hline & $>40$ years & $25(10.9 \%)$ & $54(23.5 \%)$ & & \\
\hline \multirow[t]{2}{*}{ Sex } & Male & $43(18.7 \%)$ & $57(24.8 \%)$ & \multirow{2}{*}{3.203} & \multirow{2}{*}{0.074} \\
\hline & Female & $41(17.8 \%)$ & $89(38.7 \%)$ & & \\
\hline \multirow[t]{3}{*}{ BMI } & Normal & $53(23.0 \%)$ & $89(38.7 \%)$ & \multirow[t]{3}{*}{0.106} & \multirow[t]{3}{*}{0.949} \\
\hline & Overweight & $8(3.5 \%)$ & $15(6.5 \%)$ & & \\
\hline & Underweight & $23(10.0 \%)$ & $42(18.3 \%)$ & & \\
\hline \multirow[t]{2}{*}{ Marital Status } & Unmarried/ others & $27(11.7 \%)$ & $33(14.3 \%)$ & \multirow[t]{2}{*}{2.517} & \multirow[t]{2}{*}{0.113} \\
\hline & Married & $57(24.8 \%)$ & $113(49.1 \%)$ & & \\
\hline \multirow{7}{*}{$\begin{array}{l}\text { Highest level of } \\
\text { education }\end{array}$} & Graduate & $12(5.2 \%)$ & $19(8.3 \%)$ & \multirow[t]{7}{*}{8.297} & \multirow[t]{7}{*}{0.217} \\
\hline & Higher secondary & $15(6.5 \%)$ & $20(8.7 \%)$ & & \\
\hline & Secondary & $19(8.3 \%)$ & $19(8.3 \%)$ & & \\
\hline & Lower secondary & $13(5.7 \%)$ & $25(10.9 \%)$ & & \\
\hline & Primary & $10(4.3 \%)$ & $28(12.2 \%)$ & & \\
\hline & Less than primary & $7(3.0 \%)$ & $24(10.4 \%)$ & & \\
\hline & Illiterate & $8(3.5 \%)$ & $11(4.8 \%)$ & & \\
\hline \multirow{2}{*}{$\begin{array}{l}\text { Number of family } \\
\text { members }\end{array}$} & $\leq 4$ & $32(13.9 \%)$ & $45(19.6 \%)$ & \multirow[t]{2}{*}{1.267} & \multirow[t]{2}{*}{0.260} \\
\hline & $>4$ & $52(22.6 \%)$ & $101(43.9 \%)$ & & \\
\hline \multirow{3}{*}{$\begin{array}{l}\text { Monthly family } \\
\text { income (Taka) }\end{array}$} & $<20000$ & $22(9.6 \%)$ & $36(15.7 \%)$ & \multirow[t]{3}{*}{0.121} & \multirow[t]{3}{*}{0.942} \\
\hline & $20000-40000$ & $45(19.6 \%)$ & $78(33.9 \%)$ & & \\
\hline & $>40000$ & $17(7.4 \%)$ & $32(13.9 \%)$ & & \\
\hline \multirow{2}{*}{ Sleep duration } & $\leq 7$ hours & $30(13.0 \%)$ & $63(27.4 \%)$ & 1.224 & 0.269 \\
\hline & $>7$ hours & $54(23.5 \%)$ & $83(36.1 \%)$ & & \\
\hline Walking habit (more & Yes & $25(10.9 \%)$ & $80(34.8 \%)$ & 13.467 & $0.000 *$ \\
\hline than $10 \mathrm{~min}$ at a time) & No & $59(25.7 \%)$ & $66(28.7 \%)$ & & \\
\hline Exercise habit (Other & Yes & $21(9.1 \%)$ & $71(30.9 \%)$ & 12.406 & $0.000^{*}$ \\
\hline than walking) & No & $63(27.4 \%)$ & $75(32.6 \%)$ & & \\
\hline Smoking habit & Yes & $41(17.8 \%)$ & $38(16.5 \%)$ & 12.273 & $0.000 *$ \\
\hline & No & $43(18.7 \%)$ & $108(47.0 \%)$ & & \\
\hline Number of medicine & One & $43(18.7 \%)$ & $75(32.6 \%)$ & 0.027 & 0.987 \\
\hline & Two & $39(17.0 \%)$ & $68(29.6 \%)$ & & \\
\hline & Three or more & $2(0.9 \%)$ & $3(1.3 \%)$ & & \\
\hline Presence of other & Yes & $19(8.3 \%)$ & $33(14.3 \%)$ & 0.000 & 0.998 \\
\hline chronic diseases & No & $65(28.3 \%)$ & $113(49.1 \%)$ & & \\
\hline Duration of RHD & $\leq 15$ years & $82(35.7 \%)$ & $126(54.8 \%)$ & 7.896 & $0.005^{*}$ \\
\hline & $>15$ years & $2(0.9 \%)$ & $20(8.7 \%)$ & & \\
\hline
\end{tabular}

We have identified the mean adherence of study participants was $69.41 \%$. Among them, $36.5 \%$ of participants' demonstrated low adherence to medication and $63.5 \%$ medium adherence. Nobody showed high adherence to medication.

We have used the chi-square test to describe individual variables that are associated with medication adherence. The analysis shows that, walking habit (more than ten minutes at a time) $(\mathrm{P}=0.000)$, exercise habit (Other than walking) $(\mathrm{P}=0.000)$, smoking habit $(\mathrm{P}=0.000)$, Duration of RHD $(\mathrm{P}=0.005)$ statistically significantly associated with medication adherence at 5\% significance level. Besides, these female were found more therapeutic compliant than men. Patients having more than four family members are also found more adherent than having 4 or fewer family members (Table 1 ). 
Table 2. Multiple logistic regression analysis of outcome variable (Therapeutic adherence) and characteristics of Rheumatic heart disease patients.

\begin{tabular}{|c|c|c|c|c|}
\hline Variables & Category & Estimate & Adjusted Odds ratio (95\% CI) & P-Value \\
\hline \multirow[t]{3}{*}{ Sex } & $>40$ years & & Reference & \\
\hline & Male & 0.637 & $1.890(0.904-3.951)$ & 0.089 \\
\hline & Female & & Reference & \\
\hline \multirow[t]{3}{*}{ BMI } & Normal & -0.173 & $0.841(0.381-1.859)$ & 0.669 \\
\hline & Overweight & 0.170 & $1.185(0.353-3.979)$ & 0.784 \\
\hline & Underweight & & Reference & \\
\hline \multirow[t]{2}{*}{ Marital Status } & Unmarried/ others & 0.653 & $1.921(0.883-4.179)$ & 0.097 \\
\hline & Married & & Reference & \\
\hline \multirow{7}{*}{$\begin{array}{l}\text { Highest level of } \\
\text { education }\end{array}$} & Graduate & -0.588 & $0.556(0.172-1.791)$ & 0.325 \\
\hline & Higher secondary & -1.004 & $0.366(0.110-1.222)$ & 0.102 \\
\hline & Secondary & & Reference & \\
\hline & Lower secondary & -0.747 & $0.474(0.162-1.390)$ & 0.174 \\
\hline & Primary & -1.232 & $0.292(0.093-0.915)$ & $0.035^{*}$ \\
\hline & Less than primary & -1.689 & $0.185(0.050-0.678)$ & $0.011^{*}$ \\
\hline & Illiterate & -0.262 & $0.769(0.196-3.022)$ & 0.707 \\
\hline \multirow{2}{*}{$\begin{array}{l}\text { Number of family } \\
\text { members }\end{array}$} & $\leq 4$ & 0.480 & $1.616(0.793-3.294)$ & 0.187 \\
\hline & $>4$ & & Reference & \\
\hline \multirow{3}{*}{$\begin{array}{l}\text { Monthly family income } \\
\text { (Taka) }\end{array}$} & $<20000$ & -0.078 & $0.925(0.350-2.445)$ & 0.875 \\
\hline & $20000-40000$ & -0.223 & $0.800(0.338-1.898)$ & 0.613 \\
\hline & $>40000$ & & Reference & \\
\hline \multirow{2}{*}{ Sleep duration } & $\leq 7$ hours & -0.448 & $0.639(0.321-1.270)$ & 0.199 \\
\hline & $>7$ hours & & Reference & \\
\hline \multirow{2}{*}{$\begin{array}{l}\text { Walking habit (more } \\
\text { than ten minutes at a } \\
\text { time) }\end{array}$} & Yes & & Reference & \\
\hline & No & 0.882 & $2.416(1.212-4.816)$ & $0.012^{*}$ \\
\hline \multirow{2}{*}{$\begin{array}{l}\text { Exercise habit (Other } \\
\text { than walking) }\end{array}$} & Yes & & Reference & \\
\hline & No & 0.884 & $2.420(1.206-4.859)$ & $0.013^{*}$ \\
\hline \multirow[t]{2}{*}{ Smoking habit } & Yes & & Reference & \\
\hline & No & -0.936 & $0.392(0.183-0.841)$ & $0.016^{*}$ \\
\hline \multirow[t]{3}{*}{ Number of medicine } & One & 0.745 & $2.106(0.268-16.534)$ & 0.479 \\
\hline & Two & 0.744 & $2.105(0.266-16.627)$ & 0.480 \\
\hline & Three or more & & Reference & \\
\hline \multirow{2}{*}{$\begin{array}{l}\text { Presence of other } \\
\text { chronic diseases }\end{array}$} & Yes & & Reference & \\
\hline & No & -0.256 & $0.774(0.340-1.761)$ & 0.541 \\
\hline \multirow{2}{*}{ Duration of RHD } & $<15$ years & 1.433 & $4.190(0.851-20.631)$ & $0.048^{*}$ \\
\hline & $\geq 15$ years & & Reference & \\
\hline
\end{tabular}

We have fitted a multiple logistic regression model to see the strength of association and significant predictors of low medication adherence. Very similar results observed in our adjusted model and all significant predictors of the chi-square analysis remained statistically significant. Patients don't have a walking habit (more than ten minutes at a time) have a higher risk of being low adherent to medication ( $\mathrm{AOR}=2.416,95 \% \mathrm{CI}: 1.212-4.816$ ). Similarly, patients not having an exercise habit (Other than walking) (AOR=2.420, 95\% CI: $1.206-4.859$ ), having the habit of smoking also have a higher risk of being low adherent to medication. Moreover, Patients having RHD for less than 15 years are found less adherent to medication (AOR=4.190, 95\% CI: $0.851-20.631$ ) (Table 2).

\section{Discussion}

Previously, many studies have been evolved to determine the level of therapeutic compliance among rheumatic heart disease patients (de Dassel et al., 2017; Engelman et al., 2016). Adherence usually driven by multiple factors, and best understood using a system approach considering socioeconomic, healthcare, condition, therapy, and patient-related factors (WHO, 2003). A wide range of variables targetted in these studies makes comparison particularly tricky and complicated, moreover highlights the necessity of standardized indicators to evaluate adherence. In our study, the mean adherence to medication was $69.41 \%$, which is higher than another study conducted in an aboriginal community in Australia (Stewart et al., 2007). Here, 36.5\% of participants 
demonstrated low adherence to medication and $63.5 \%$ medium adherence. Nobody showed high adherence to medication. These findings are comparable to some other studies conducted in different parts of the world and Bangladesh (Engelman et al., 2016; Gasse et al., 2013; Islam and Majumder, 2016).

Our result shows that walking habit (more than ten minutes at a time) $(\mathrm{P}=0.000)$, exercise habit (Other than walking) $(\mathrm{P}=0.000)$, smoking habit $(\mathrm{P}=0.000)$, Duration of $\mathrm{RHD}(\mathrm{P}=0.005)$ statistically significantly associated with medication adherence. Patients with higher diagnosis duration $(\geq 15)$ were more likely to be adherent compared to patients with lower diagnosis duration $(<15)$. This outcome was similar to the studies conducted in Canada (Kholdebarin et al., 2008), USA (Boland et al., 2014; Dreer et al., 2012), and Germany (Welge-Lussen et al., 2015). Our result demonstrated that walking habit and exercise habit other than walking has a significant association with medication adherence, from our perspective the reason could be very simple but elegant, which is, people who regularly exercise or at least walk regularly to keep them healthy are concerned about their health. This ultimately leads them to proper uses and maintenance of their daily or diseased based medication, which ends with having a higher percentage of adherence towards their treatment. On the flip side, people with the habit of smoking is also strongly associated with low medication adherence, and they usually tend to care less about their health. This one habit of smoking exhibits that people with a habit of smoking have lesser concerned about their health and uses of their medication which was found in a study that was done in Appalachia in 2010 (Basta et al., 2012). Which in the end, comes down to a low percentage of adherence to medication.

\section{Conclusions}

In our study, we have found medium to a low level of medication adherence among patients with rheumatic heart disease. There are various factors like walking habit, exercise habit, smoking habit, and duration of RHD have a strong association with medication adherence. So from the findings of our results, we can confidently say that if our healthcare providers take these associated factors into their consideration and discuss on these factors with their patients, before giving their patients any treatment will surely help to improve medication adherence among rheumatic heart disease patients. These findings highlight to seek for possible future research and studies to identify and to understand the mechanisms of underlying lower adherence to the medication of rheumatologic heart disease. Therefore, it will create the opportunity to discover an improved intervention for patients with RHD as well as the chance to enhance medication adherence in RHD patients.

\section{Conflict of interest}

None to declare.

\section{References}

Basta T, A Bansah and HD Holben, 2012. Relationship between smoking and medication adherence among individuals living with HIV in rural Appalachia. 140st APHA Annual Meeting and Exposition. Retrieved from

https://www.researchgate.net/publication/266776958_Relationship_between_smoking_and_medication_adh erence_among_individuals_living_with_HIV_in_rural_Appalachia

Berhe DF, K Taxis, FM Haaijer-Ruskamp, A Mulugeta, YT Mengistu, JGM Burgerhof and PGM Mol, 2017. Impact of adverse drug events and treatment satisfaction on patient adherence with antihypertensive medication - a study in ambulatory patients. Br. J. Clin. Pharmacol., 83: 2107-2117.

Boland MV, DS Chang, T Frazier, R Plyler and DS Friedman, 2014. Electronic Monitoring to Assess Adherence With Once-Daily Glaucoma Medications and Risk Factors for Nonadherence. JAMA Ophthalmology, 132: 838.

Busse R, D Scheller-kreinsen and A Zentner, 2010. Tackling Chronic: Strategies, interventions and challenges. European Observatory on Health Systems and Policies.

Carapetis JR, BM Mayosi and EL (n.d.) Kaplan, 2013. Controlling rheumatic heart disease in developing countries. Cardiovascular Journal of South Africa, 17: 164-165.

Carapetis JR, AC Steer, EK Mulholland and M Weber, 2005. The global burden of group A streptococcal diseases. Lancet Infect. Dis., 5: 685-694.

de Dassel JL, MT Fittock, SC Wilks, JE Poole, JR Carapetis and AP Ralph, 2017. Adherence to secondary prophylaxis for rheumatic heart disease is underestimated by register data. PLOS ONE, 12: e0178264.

Dreer LE, C Girkin and SL Mansberger, 2012. Determinants of medication adherence to topical glaucoma therapy. Journal of Glaucoma, 21: 234-240.

Elliott RA and JL Marriott, 2009. Standardised assessment of patients' capacity to manage medications: a 
systematic review of published instruments. BMC Geriatrics, 9: 27.

Engelman D, RL Mataika, JH Kado, M Ah Kee, S Donath, T Parks and AC Steer, 2016. Adherence to secondary antibiotic prophylaxis for patients with rheumatic heart disease diagnosed through screening in Fiji. Trop. Med. Int. Health, 21: 1583-1591.

Gasse B, N Baroux, B Rouchon, JM Meunier, IDe Frémicourt and E D'Ortenzio, 2013. Determinants of poor adherence to secondary antibiotic prophylaxis for rheumatic fever recurrence on Lifou, New Caledonia: a retrospective cohort study. BMC Public Health, 13: 131.

Islam AKMM and AAS Majumder, 2016. Rheumatic fever and rheumatic heart disease in Bangladesh: A review. Indian Heart J., 68: 88-98.

Karthikeyan G, L Zühlke, M Engel, S Rangarajan, S Yusuf, K Teo and BM Mayosi, 2012. Rationale and design of a Global Rheumatic Heart Disease Registry: The REMEDY study. Am. Heart J., 163: 535-540.

Kholdebarin R, RJ Campbell, YP Jin and YM Buys, 2008. Multicenter study of compliance and drop administration in glaucoma. Can. J. Ophthalmol., 43: 454-461.

Lawrence JG, JR Carapetis, K Griffiths, K Edwards and JR Condon, 2013. Acute rheumatic fever and rheumatic heart disease: incidence and progression in the Northern Territory of Australia, 1997 to 2010. Circulation, 128: 492-501.

Marijon E, M Mirabel, DS Celermajer and X Jouven, 2012. Rheumatic heart disease. The Lancet, 379: 953964.

Morisky DE and LW Green, 1986. Concurrent and predictive validity of a self-reported measure of medication adherence: Morisky 8-Item Medication Adherence Questionnaire. Med. Care, 24: 67-74.

Nulu S, G Bukhman and GF Kwan, 2017. Rheumatic Heart Disease. Cardiology Clinics, 35: 165-180.

Oldgren J, JS Healey, M Ezekowitz, P Commerford, A Avezum, P Pais,... RE-LY Atrial Fibrillation Registry Investigators, 2014. Variations in cause and management of atrial fibrillation in a prospective registry of 15,400 emergency department patients in 46 countries: the RE-LY Atrial Fibrillation Registry. Circulation, 129: $1568-1576$.

Paar JA, NM Berrios, JD Rose, M Cáceres, R Peña, W Pérez,... JB Dale, 2010. Prevalence of Rheumatic Heart Disease in Children and Young Adults in Nicaragua. Am. J. Cardiol., 105: 1809-1814.

Sokol MC, KA McGuigan, RR Verbrugge and RS Epstein, 2005. Impact of medication adherence on hospitalization risk and healthcare cost. Medical Care, 43: 521-530.

Stewart T, R McDonald and B Currie, 2007. Acute rheumatic fever: Adherence to secondary prophylaxis and follow up of Indigenous patients in the Katherine region of the Northern Territory. Australian Journal of Rural Health, 15: 234-240.

Welge-Lussen U, S Weise and AL Yu, 2015. Assessing the adherence behavior of glaucoma patients to topical eye drops. Patient Preference and Adherence, 9: 17-23.

WHO, 2003. Adherence to long-term therapies: evidence for action. Retrieved from https://apps. who.int/iris/bitstream/handle/10665/42682/9241545992.pdf;jsessionid=DA3D882B8F73091259 C7DB59DA9E04EC? sequence=1

Wilke T, S Müller and DE Morisky, 2011. Toward Identifying the Causes and Combinations of Causes Increasing the Risks of Nonadherence to Medical Regimens: Combined Results of Two German Self-Report Surveys. Value in Health, 14: 1092-1100. 